\title{
Estudio dinámico de la movilidad escolar mediante tecnologías web de geolocalización
}

\author{
Varela-García, Francisco-Alberto \\ cartoLAB, Grupo de Visualización Avanzada e Cartografía, Departamento de Métodos \\ Matemáticos y de Representación, ETS Ingeniería de Caminos, Canales y Puertos, \\ Universidade da Coruña, Campus de Elviña, 15071 A Coruña, España
}

\section{Hernández Ibáñez, Luis}

videaLAB, Grupo de Visualización Avanzada e Cartografía, Departamento de Métodos Matemáticos y de Representación, ETS Ingeniería de Caminos, Canales y Puertos, Universidade da Coruña, Campus de Elviña, 15071 A Coruña, España

\section{Novales Ordax, Margarita}

Grupo de Ferrocarriles y Transportes, Departamento de Métodos Matemáticos y de Representación, ETS Ingeniería de Caminos, Canales y Puertos, Universidade da Coruña, Campus de Elviña, 15071 A Coruña, España

\section{Orro Arcay, Alfonso}

Grupo de Ferrocarriles y Transportes, Departamento de Métodos Matemáticos y de Representación, ETS Ingeniería de Caminos, Canales y Puertos, Universidade da Coruña, Campus de Elviña, 15071 A Coruña, España

\section{Gutiérrez Puebla, Javier}

Grupo de Transporte, Infraestructuras y Territorio, Departamento de Geografía Humana, Facultad de Geografía e Historia, Universidad Complutense de Madrid, Ciudad Universitaria, 28040 Madrid, España

\section{López Fernández, Jorge}

cartoLAB, Grupo de Visualización Avanzada e Cartografía, Departamento de Métodos Matemáticos y de Representación, ETS Ingeniería de Caminos, Canales y Puertos, Universidade da Coruña, Campus de Elviña, 15071 A Coruña, España

\section{RESUMEN}

Los desplazamientos realizados a los centros de enseñanza son unos de los comportamientos de movilidad más sensibles debido a la vulnerabilidad de sus principales protagonistas: los niños, y su interacción con otros tipos de usuarios. Además, la temporalidad de estos movimientos es un factor de alto impacto, pues provoca problemas puntuales de congestión en zonas próximas a los colegios en las horas concretas de entrada y salida, por lo que se generan conflictos en la circulación, poniendo en riesgo a usuarios, y a conductores y peatones del entorno.

El domicilio habitual de los alumnos y sus modos de desplazamiento hasta el centro 
educativo, serán datos fundamentales para poder establecer políticas adecuadas de movilidad en cada uno de los centros. Y para poder formular acciones conjuntas en esta materia sobre una región, se requiere conocer con rigurosidad, y de una forma global, el problema existente en un gran número de centros. En este trabajo se desarrolla una investigación sobre seguridad vial en entornos escolares, basada en las nuevas tecnologías de información y comunicación, desarrollando y aplicando técnicas de geolocalización a través de Internet, que permiten relacionar datos sobre el comportamiento de los usuarios de los viales, con las localizaciones del origen y destino de los desplazamientos. De esta manera se dispone de información desagregada de gran interés para analizar sobre Sistemas de Información Geográfica (SIG), estudiando rutas óptimas desde domicilios a centros educativos, viales empleados, longitudes de recorridos, características de los desplazamientos, adecuación de infraestructuras, modos y servicios de transporte, etc., con lo que se elabora un complejo informe estadístico sobre la movilidad escolar.

Este trabajo cuenta con una subvención de la DGT para el desarrollo de proyectos de investigación en el área de tráfico, movilidad y seguridad vial correspondientes a 2015.

\section{INTRODUCCIÓN}

Una de las prioridades de la Estrategia de Seguridad Vial 2011-2020 de la DGT es la protección de los usuarios más vulnerables, entre la que se encuentran los niños, los mayores, los ciclistas y los peatones. Todos estos colectivos están presentes en los desplazamientos realizados a los centros de enseñanza, que además debido a sus especiales características, son uno de los comportamientos de movilidad más sensibles en cuanto a la seguridad vial. En primer lugar por la vulnerabilidad de los protagonistas principales: los niños. Pero también el resto de usuarios que intervienen en estos desplazamientos, como son muchas veces los mayores, abuelos o familiares de los niños, que los acompañan en su recorrido, como pone de manifiesto el estudio publicado por Audi Attitudes en colaboración con el INTRAS, donde indica que el $70 \%$ de los niños españoles no van solos al colegio(Alonso, Esteban, Calatayud, \& Alamar, 2009). En ese trabajo, donde las respuestas a los modos de desplazamiento usados pueden ser múltiples (por lo que la suma no es del 100\%), se muestra como un $11 \%$ optan por el transporte escolar o público, un $40 \%$ por el vehículo privado, y la gran mayoría, el 59\% van andando, sobre todo en municipios pequeños.

El importante número de desplazamientos escolares a pie, en convivencia con otros medios de transporte, añade un factor más de riesgo ante accidentes de tráfico, que se unen además a la temporalidad de estos movimientos, concentrados en las horas de entrada y salida de las actividades escolares, provocando problemas puntuales de congestión en zonas próximas a los centros de enseñanza, y conflictos diversos en la circulación, que ponen en riesgo a conductores y peatones. Si unimos a todo ello, la participación de transporte privado, transporte colectivo, tanto público como propio del centro escolar, la existencia de usuarios 
en bicicleta, junto con el tráfico peatonal, observamos como los desplazamientos escolares generan una movilidad muy particular que debe ser atendida con un enfoque especial, frente a otro tipo de actividades generadoras de tráfico.

El conocimiento de la ubicación de los alumnos será fundamental para poder establecer políticas adecuadas de movilidad en cada uno de los centros. Disponer de estos datos supone indirectamente, saber los recorridos que deben realizar, y los viales que deben usar, pudiendo valorar la adecuación de los mismos al nivel de flujos que van a tener que soportar, y analizar si cumplen los requisitos para caracterizarlos como "caminos escolares seguros" que la DGT está intentando promocionar junto con la Federación de Municipios y Provincias en aquellos colegios interesados en fomentar una movilidad sostenible desde edades tempranas. Además, disponer de datos de concentración de alumnos en determinadas zonas o a través de trayectos concretos, puede ofrecer información relevante para planificar rutas de transporte colectivo, tanto públicas como propias de los centros. Aunque normalmente existes unos criterios de admisión en donde se prima la cercanía al centro escolar, otras circunstancias pueden sobreponerse a ese criterio, por lo que no es extraño encontrar que muchos de los alumnos de colegios e institutos tengan su residencia en lugares distantes a estos centros. En el transporte escolar, suelen existir rutas definidas históricamente sin ser sometidas a un proceso de optimización en función de la ubicación de los usuarios demandantes, lo que aumenta la carga económica para las administraciones regionales que sostienen este tipo de transporte.

Conseguir el domicilio de todos los alumnos de un centro, es relativamente sencillo para los responsables del centro, y también para las autoridades educativas de una Comunidad Autónoma. Sin embargo, trasladar esos domicilios a un Sistema de Información Geográfica (SIG) que permita su análisis integrado con los datos del viario y de otras informaciones territoriales, requiere de procesos de geoposicionamiento con una complejidad que restringe su utilización para personal no suficientemente formado, además de los criterios de protección de datos que deben cumplirse. La mayoría de las encuestas realizadas para conocer la movilidad de una población se basan en referencias geográficas muy genéricas (a nivel municipal, por ejemplo), pero muy pocas trabajan con la ubicación real y detallada de los orígenes y destinos de los usuarios, pues requieren importantes recursos para su adecuado a análisis. En muchas ocasiones simplemente se trabaja con la combinación de estimaciones de tiempos de viaje y distancias, como manifiestan (Lardelli Claret et al., 2012), en su proyecto de diseño de un cuestionario sobre movilidad.

Las nuevas tecnologías de información y comunicación ofrecen infinidad de nuevas posibilidades a los ciudadanos y a las administraciones, que con mayor o menor acierto, y con más o menos celeridad, van descubriendo y aplicando a sus actividades. Pero estas nuevas tecnologías también ofrecen servicios que muchos investigadores de temáticas muy variadas podrían usar en sus proyectos, para obtener datos de enorme valor en sus investigaciones, y que muchas veces serían imposible de conseguir, o resultarían demasiado 
costosos por medios tradicionales. Este es el caso de las técnicas web de geolocalización, con las que el equipo del laboratorio de cartografía (cartoLAB) del Grupo de Visualización Avanzada e Cartografía de la Universidade da Coruña (UDC) lleva trabajando desde hace tiempo para elaborar encuestas geolocalizadas con las que fácilmente se consigue, no sólo de las respuestas de los encuestados a un número de preguntas, sino también su localización geográfica precisa, con la que poder posteriormente realizar los análisis geoespaciales necesarios. Actualmente se cuenta con una aplicación en modo alpha de esta tecnología en la web https://emapic.es, que puede ser empleado por cualquier interesado para todo tipo de temáticas.

La elaboración de encuestas geolocalizadas es una tecnología altamente innovadora, y no hay en el ámbito científico ni comercial referencias a la aplicación de la geolocalización web en estudios de movilidad, por lo que esta investigación supone un aspecto innovador al asociar localizaciones origen con los destinos de los desplazamientos para análisis más profundos de los resultados de las encuestas tradicionales.

La elaboración de este proyecto necesita de dos aspectos cruciales para conseguir los resultados esperados: desarrollar la tecnología que permita la realización de encuestas geolocalizadas, y conseguir una muestra representativa de encuestados pertenecientes a colegios e instituciones educativas para que respondan sobre cuestiones relativas a la movilidad diaria a sus centros escolares. Ambos aspectos serán tratados brevemente en los siguientes apartados.

\section{TECNOLOGÍA WEB DE GEOLOCALIZACIÓN}

La implementación y desarrollo de una tecnología web de encuestas geolocalizadas en donde la referencia geográfica sea un aspecto fundamental de las mismas, es una de las premisas de este proyecto. Los trabajos desarrollados desde el cartoLAB, implementados dentro de la aplicación EMAPIC, permiten disponer de un visor web de mapas multiparamétrico, que permite identificar la localización geográfica de los colegios, y al mismo tiempo recoge la ubicación del usuario gracias a diferentes estrategias de localización, manual y/o automática. Se establece un proceso de vinculación entre las respuestas de la encuesta de movilidad y el centro escolar escogido por el usuario, recogiendo todos los datos para su análisis posterior, y mostrando ciertos datos de forma abierta para conocer el grado de participación de cada centro, en comparación con otros, durante la elaboración del proyecto para fomentar su colaboración.

La aplicación web implementada se desarrolla empleando un conjunto de tecnologías HTML5, CSS3 y Javascript, para facilitar la instalación, y flexibilizar la disponibilidad de la aplicación desde cualquier navegador web común, y su utilización prácticamente desde cualquier multiplataforma, pudiendo ejecutarse desde distintos sistemas operativos. Para la 
representación geográfica, la aplicación consta de un visor geográfico realizado con la librería Javascript de código abierto Leaflet, que emplea HTML5 y CSS3 para representación de mapas interactivos en web. El visor cuenta con una serie de elementos de interacción con el mapa como herramientas de visualización y zoom, para ofrecer una gran facilidad de uso a cualquier persona usuaria de un navegador web, sin que necesite conocimientos previos adicionales. Las capas cartográficas de base mostradas en el visor son de OpenStreetMap (OSM) y de Mapbox, y sobre ellas se muestran los colegios participantes en el proyecto, y sirve también para mostrar la ubicación de cada encuestado mientras realiza la encuesta. Además este mismo visor nos sirve al equipo de investigación para mostrar todas las encuestas vinculadas a cada colegio, y hacer análisis pormenorizado de todas las respuestas.

Esta aplicación para la generación de encuestas geolocalizadas, toma las coordenadas y datos propios asociados referentes a los centros educativos de archivos con formato geojson (una variante del formato JSON - JavaScript Object Notation - para el almacenamiento de objetos Javascript en texto plano, pero que además incluye información espacial), y los resultados obtenidos en las consultas realizadas a los diferentes encuestados (tanto las coordenadas de su ubicación geográfica, como las respuestas contestadas) se almacenan en una base de datos PostgreSQL.

La ubicación geográfica del encuestado, que debería referenciarse aproximadamente con el domicilio del mismo, se puede obtener automáticamente a partir de los servicios de localización disponibles en los navegadores, pero también se deja la posibilidad de realizar esta ubicación manualmente por parte los usuarios mediante la identificación sobre el mapa del lugar correcto, modificando el resultado de la localización automática, o bien corrigiendo esta posición para ser lo más preciso posible. Los servicios basados en la localización automática por parte de los dispositivos informáticos de los usuarios, se fundamentan en diferentes estrategias (Koch, Golling, \& Rodosek, 2013) tanto utilizando la propia red de comunicaciones por cable, GSM o WiFi, aunque dependiendo de las aplicaciones se pueden atender también a la infraestructura GPS. Bajo el acrónimo de SOLOMO (Social, Local y Móvil) se agrupan gran parte de las estrategias de desarrollo de Internet en nuestros días (Reed, 2011), fundamentalmente enfocados al marketing para utilizar la localización de los potenciales clientes o usuarios, aunque debemos considerar la fiabilidad en la precisión ofrecida que algunos autores cuestionan (Backstrom, Sun, \& Marlow, 2010; Poese, Uhlig, Kaafar, Donnet, \& Gueye, 2011; Shavitt, Member, \& Zilberman, 2011). De hecho, un aspecto de interés de este proyecto, es valorar la precisión que ofrecen los servicios de geolocalización basados en web, para cuantificar hasta que escala territorial pueden ser tenidos en cuenta en estudios de movilidad y de accesibilidad, los datos suministrados por este tipo de procedimientos. 


\section{CENTROS ESCOLARES E INSTITUCIONES COLABORADORAS}

Para que el estudio de movilidad escolar pueda realizar, es necesario que las diferentes encuestas geolocalizadas preparadas sean cubiertas por el perfil social necesario. Se elabora una encuesta para niños de Educación Infantil (de 3 a 5 años) y de Primaria de entre 5 y 8 años (cursos $1^{\circ}, 2^{\circ}, 3^{\circ}$ y $4^{\circ}$ ), que tendrán una parte específica para sus padres; también se crea un cuestionario específico para los cursos $5^{\circ}$ y $6^{\circ}$ de Primaria con el objetivo de que puedan ser ellos mismos los que cubran la encuesta con el apoyo de sus profesores o de sus padres en caso de que no puedan cubrirla en el colegio por falta de aulas informáticas adecuadas. Además se desarrolla un cuestionario más completo para adolescentes que estudien ESO, Bachiller o Ciclos Formativos, en donde el grado de complejidad de las preguntas aumenta, y se busca una interacción más activa de los alumnos con el mapa, llegando incluso a solicitar que identifiquen lugares problemáticos en sus recorridos, o marquen puntos de paso para trazar sus itinerarios y disponer así de mayor información para estudiar y analizar la movilidad de cada centro.

Para llevar a cabo estas encuestas se establece contactos con centros educativos interesados, bien directamente, o a través de proyectos vinculados con el establecemiento de caminos escolares seguros, impulsados desde la propia DGT, la Consellería de Cultura, Educación e Ordenación Universitaria de la Xunta de Galicia, especialmente a trvés de su programa PROXECTA, desde Ayuntamientos como por ejemplo el de A Coruña, Ferrol y Narón, y también desde asociaciones especializadas en la movilidad, destacando el grado de implicación de Stop Accidentes. La población a encuestar por tanto corresponde con los alumnos de infantil, primaria, secundaria, ciclos y bachillerato de los centros escolares que se adhieren a este proyecto. Es necesario que un centro confirme su interés en participar para poder iniciar el proceso de realización de la encuesta por parte de sus alumnos.

La aplicación tecnológica desarrollada permite realizar la encuesta a un número ilimitado de alumnos y de centros, y esta es una de sus grandes fortalezas, pues no es necesario implementar recursos adicionales si la encuesta se amplía a una mayor cantidad de encuestados. Sin importar además su localización geográfica, o al menos sin que sea un problema significativo para poder abordarse, pues no es necesario desplazar ni técnicos ni encuestadores para poder realizarse. En una primera fase de contactos se llega casi 30 colegios de Galicia que se acogen a esta iniciativa, pero el proyecto está aún en desarrollo durante todo este 2016, con lo que se pretende llegar a más centros, incluso de fuera de la comunidad gallega.

El diseño de los cuestionarios, aunque cada uno con un nivel de intensidad y complejidad diferente según el grado educativo al que se destine, pregunta por el modo de transporte usualmente empleado para el desplazamiento al centro escolar, el tiempo de viaje empleado, el sistema de seguridad del vehículo usado, el conocimiento o involucración en algún 
accidente en este recorrido, las características sociales de la familia, etc. Además del análisis estadístico de estos parámetros, se estudia su relación con las características territoriales existentes en cada centro, el tipo de viario de las rutas posibles en los desplazamientos hasta cada centro educativo, etc., y con ello se relacionan las respuestas con su localización con respecto al centro y al resto de elementos de su entorno. Los datos del viario se obtienen a partir de diferentes bases de datos geográficas disponibles por parte de diferentes administraciones públicas, tanto nacionales, como autonómicas, provinciales y locales.

La preparación de la encuesta, la confirmación de los centros a encuestar y el desarrollo de la encuesta se realiza durante los 3-4 primeros meses del proyecto para así conseguir la máxima participación posible en los siguientes meses del proyecto, que durará todo el 2016, y obtener así las mejores garantías de calidad al poder conseguir la mayor cantidad de respuestas posibles.

\section{RESULTADOS Y CONCLUSIONES}

Puesto que el proyecto aún está en desarrollo sólo se disponen de datos parciales que deben ir completándose durante los próximos meses. Es pronto aún para mostrar resultados concretos y conclusiones definitivas del estudio, pero con los datos que se van obteniendo en la aplicación desarrollada, se estudia con un grado de detalle importante la movilidad existente en los centros educativos adheridos al proyecto, especialmente en la Comunidad Autonómica de Galicia. Las especiales características orográficas, climatológicas y de distribución poblacional de la comunidad gallega, hace interesante profundizar en su sistema de transporte y movilidad escolar, con la finalidad de encontrar patrones de comportamiento, y características concretas de su aplicación que puedan ser mejorados. Pero el proyecto pretende también recopilar información de centros de otras regiones de España durante su segunda fase. Para que este análisis tenga la máxima veracidad, es importante conseguir la mayor cantidad de participantes, por lo que se realiza una campaña de concienciación sobre la movilidad sostenible en entornos escolares, que además de dar a conocer este proyecto, fomente la responsabilidad y seguridad vial desde edades tempranas. Con el objetivo de conseguir más dinamismo en la contestación al cuestionario, se ofrece una página específica donde se puede ver prácticamente en tiempo real, el grado de participación de cada centro, y algunas de las respuestas más interesantes para el público en general, como el porcentaje de uso del modo de transporte en cada caso.

El análisis de movilidad realizado en este proyecto, no se limita exclusivamente a elaborar un estudio estadístico de las variables recopiladas en la encuesta. Puesto que se dispondrá de la ubicación geográfica de cada encuestado, se realiza un ambicioso estudio de accesibilidad a cada centro escolar, analizando distancias máximas, medias, recorridos óptimos, nivel de centralidad, etc. Para realizar los cálculos de accesibilidad es fundamental el empleo de Sistemas de Información Geográfica (SIG) por lo que la información obtenida se vuelca a 
estos sistemas para realizar las operaciones de análisis de redes precisas. Para ello se utiliza una red con topología a partir de la cartografía procedente de OpenStreetMap, a través del servicio pgRouting, y también descargando los datos viarios de los ámbitos geográficos a estudiar sobre entornos SIG.

Los resultados alcanzados se pueden analizar desde dos puntos de vista: los propios datos de movilidad que se pueden conseguir, y la tecnología usada para conseguirlos.

Los datos recopilados sobre la movilidad en centros escolares, aún en estudio en el momento de redacción de este artículo, son de especial interés para conocer este tipo de actividad dentro del sistema viario. En particular interesará conocer la situación en Galicia, puesto que esta Comunidad Autónoma presenta unas condiciones geográficas y de distribución poblacional muy particulares en el conjunto de España, (sólo recordar que en Galicia se encuentra casi el 50\% de las entidades de población de todo el país, pese a tener un tamaño que apenas supone el $5 \%$ de la superficie), lo que condiciona enormemente su movilidad. Conseguir una información detallada sobre las características de los desplazamientos escolares es de gran relevancia para entender su funcionamiento, y a partir de ahí, que las autoridades tomen las medidas más oportunas para mejorarlo. En este sentido, tanto la DGT como la Xunta de Galicia están interesados en estos resultados, pero especialmente la administración local de los municipios sobre la que se consiga información. Son los propios centros educativos, y las autoridades de los municipios donde se ubican, quienes emplearán estos resultados para poder ejecutar planes de movilidad específicos, apoyados en el interés de la propia DGT, a través de sus Jefaturas provinciales de Tráfico, y la Federación de Municipios y Provincias, por incentivar los caminos escolares seguros, fomentando en aquellos colegios interesados la movilidad sostenible en edades tempranas. Con la experiencia obtenida hasta ahora, estamos convencidos de que los propios centros son los más interesados en disponer y manejar los datos obtenidos en el proyecto, tanto los propios, como los comparativos con otros centros similares.

Desde el punto de vista de la tecnología web de geolocalización utilizada en este proyecto, se ha mostrado como una metodología extrapolable a cualquier otro estudio de movilidad similar, sin importar la extensión ni el ámbito geográfico local, regional o a nivel mundial del proyecto, lo que supone una herramienta de gran potencial para disponer de una manera más o menos sencilla de un enorme volumen de datos con componente geográfica, de extraordinario interés. En este sentido, ya se está implementando su aplicación también dentro de otro proyecto de investigación, financiado en la Convocatoria 2015 del Programa Estatal de I+D+i Orientada a los Retos de la Sociedad, para elaborar una investigación titulada Análisis dinámico de accesibilidad y movilidad a partir de big data, y liderada por los investigadores Javier Gutiérrez Puebla (integrado también en el equipo de investigación de esta propuesta) y Juan Carlos García Palomares del grupo tGIS perteneciente a la Universidad Complutense de Madrid. 
Como consideración final, decir que aunque las respuestas a los cuestionarios son anónimas, se recogen ciertos datos de carácter privado, y en especial la ubicación geográfica de los encuestados. Sin embargo los datos recopilados son exclusivamente utilizados para el proyecto de investigación planteado, y en ningún momento se mostrará públicamente información individual de las encuestas realizadas.

\section{AGRADECIMIENTOS}

Este artículo se encuadra dentro del proyecto titulado ESTUDIO DINÁMICO DE LA MOVILIDAD ESCOLAR MEDIANTE TECNOLOGÍAS WEB DE

GEOLOCALIZACIÓN y que ha sido subvencionado, con la referencia SPIP2015-01867, mediante Resolución de 24 de noviembre de 2015 de la Dirección General de Tráfico, por la que se resuelve la convocatoria de subvenciones destinadas al desarrollo de proyectos de investigación en el área de tráfico, movilidad y seguridad vial, correspondientes al ejercicio 2015, convocatoria efectuada por Resolución de 1 de julio de 2015, de la Dirección General de Tráfico (B.O.E. núm. 160, de 6 de julio).

\section{REFERENCIAS}

Alonso, F., Esteban, C., Calatayud, C., \& Alamar, B. (2009). LOS NIÑNS, LAS

CIUDADES Y LA SEGURIDAD VIAL: UNA VISIÓN A PARTIR DE LA

INVESTIGACIÓN. Los niños, las ciudades la seguridad vial: Una visión a partir de la investigación. Attitudes, Audi. Retrieved from

http://attitudes.hl187.dinaserver.com/wp-

content/uploads/2009/11/Cuaderno_Reflexion_15.pdf

Backstrom, L., Sun, E., \& Marlow, C. (2010). Find me if you can: improving geographical prediction with social and spatial proximity. Proceedings of the 19th International Conference on World Wide Web, 61-70. doi:10.1145/1772690.1772698

Koch, R., Golling, M., \& Rodosek, G. D. (2013). Advanced Geolocation of IP Addresses, 7(8), 1007-1016.

Lardelli Claret, P., Jiménez, E., Pulido, J., Martínez, V., Barrio, G., Luna, J. de D., \& de la Fuente, L. (2012). INFORME FINAL DEL PROYECTO 0100DGT21262 DISEÑO DE UN CUESTIONARIO SOBRE MOVILIDAD Y EXPOSICIÓN AL TRÁFICO RODADO . PROYECTO DE APLICACIÓN. Todos los trabajos necesarios para realizar este proyecto han sido financiados por la Dirección General de Tráfico.

Poese, I., Uhlig, S., Kaafar, M. A., Donnet, B., \& Gueye, B. (2011). IP geolocation databases. ACM SIGCOMM Computer Communication Review, 41(2), 53. doi:10.1145/1971162.1971171 
Reed, R. (2011). The SoLoMo Manifesto or just about everything marketers need to know about the convergence of social, local and mobile, 30. Retrieved from http://momentfeed.com/customer-moments/solomo/

Shavitt, Y., Member, S., \& Zilberman, N. (2011). A Geolocation Databases Study, 29(10), 2044-2056. 